\title{
There is No EPTAS for Two-dimensional Knapsack
}

\author{
Ariel Kulik* Hadas Shachnai ${ }^{\dagger}$
}

\begin{abstract}
In the d-dimensional (vector) knapsack problem given is a set of items, each having a $d$-dimensional size vector and a profit, and a $d$-dimensional bin. The goal is to select a subset of the items of maximum total profit such that the sum of all vectors is bounded by the bin capacity in each dimension. It is well known that, unless $P=N P$, there is no fully polynomial time approximation scheme for $d$-dimensional knapsack, already for $d=2$. The best known result is a polynomial time approximation scheme (PTAS) due to Frieze and Clarke (European J. of Operational Research, 100-109, 1984) for the case where $d \geq 2$ is some fixed constant. A fundamental open question is whether the problem admits an efficient PTAS (EPTAS).

In this paper we resolve this question by showing that there is no EPTAS for $d$ dimensional knapsack, already for $d=2$, unless $W[1]=F P T$. Furthermore, we show that unless all problems in SNP are solvable in sub-exponential time, there is no approximation scheme for two-dimensional knapsack whose running time is $f(1 / \varepsilon)|\mathcal{I}|^{o(\sqrt{1 / \varepsilon})}$, for any function $f$. Together, the two results suggest that a significant improvement over the running time of the scheme of Frieze and Clarke is unlikely to exist.
\end{abstract}

Keywords: two-dimensional knapsack, efficient polynomial time approximation schemes, parameterized complexity, theory of computation

\section{Introduction}

In the well known $d$-dimensional knapsack problem, given is a set of $n$ items $\{1, \ldots, n\}$, where each item $i$ has a $d$-dimensional size vector $\bar{s}_{i} \geq 0$, and a profit $p_{i}>0$. Also, given is a $d$-dimensional bin whose capacity is $\bar{B}=\left(B_{1}, \ldots, B_{d}\right)$. A feasible solution is a subset of the items $A^{\prime} \subseteq A$ such that the total size of the items in $A^{\prime}$ in each dimension $r$ is bounded by $B_{r}$, $1 \leq r \leq \bar{d}$. The objective is to find a feasible solution of maximum total profit. The special case where $d=1$ is the classic 0-1 knapsack problem.

This paper studies the efficiency of finding $(1-\varepsilon)$-approximations for $d$-dimensional knapsack. A maximization problem $\Pi$ admits a polynomial-time approximation scheme (PTAS) if there is an algorithm $\mathcal{A}(\mathcal{I}, \varepsilon)$ such that, for any $\varepsilon>0$ and any instance $\mathcal{I}$ of $\Pi, \mathcal{A}(\mathcal{I}, \varepsilon)$ outputs a $(1-\varepsilon)$-approximate solution in time $|\mathcal{I}|^{f(1 / \varepsilon)}$ for some function $f$. As $\varepsilon$ gets smaller, the exponent of the polynomial $|\mathcal{I}|^{f(1 / \varepsilon)}$ may become very large. Two important restricted classes of approximation schemes were defined to eliminate this dependence. An efficient polynomialtime approximation scheme (EPTAS) is a PTAS whose running time is $f(1 / \varepsilon)|\mathcal{I}|^{O(1)}$, whereas a fully polynomial time approximation scheme (FPTAS) runs in time $(1 / \varepsilon)^{O(1)}|\mathcal{I}|^{O(1)}$.

\footnotetext{
${ }^{*}$ Computer Science Dept., Technion, Haifa 32000, Israel. E-mail: kulik@cs.technion.ac.il.

${ }^{\dagger}$ Computer Science Dept., Technion, Haifa 32000, Israel. E-mail: hadas@cs.technion.ac.il. Work supported by the Technion V.P.R. Fund.
} 
While the classic 0-1 knapsack problem admits an FPTAS, i.e., for any $\varepsilon>0$, a $(1-\varepsilon)$ approximation for the optimal solution can be found in $O\left(n / \varepsilon^{2} \cdot \log (1 / \varepsilon)\right)$ steps $[10,11],{ }^{1}$ packing in higher dimensions (also known as d-dimensional vector packing) is substantially harder to solve, exactly or approximately. It is well known that, unless $P=N P$, there is no FPTAS for $d$-dimensional knapsack, already for $d=2[12,14]$ (see also [13],[7]). Frieze and Clarke developed in $[6]$ the first PTAS for the $d$-dimensional knapsack. Subsequently, a scheme with improved running time of $O\left(n^{\lceil d / \varepsilon\rceil-d}\right)$ was given by Caprara et al. [1].

As $d$-dimensional knapsack does not admit an FPTAS, a fundamental open question is whether there exists an EPTAS. In this paper we resolve this question by showing that there is no EPTAS for two-dimensional knapsack, unless $W[1]=F P T .^{2}$ Furthermore, we use the results of [2] to show that unless all problems in SNP are solvable in sub-exponential time ${ }^{3}$ there is no approximation scheme for two-dimensional knapsack whose running time is $f(1 / \varepsilon)|\mathcal{I}|^{o(\sqrt{1 / \varepsilon})}$, for any function $f$. Together, the two results suggest that a significant improvement over the running time of the scheme of [1] is unlikely to exist. We note that, for the case where $d=1$ an EPTAS exists also for the multiple knapsack problem (see the recent work of Jansen [9]).

\section{Hardness Results}

Denote by $O P T(\mathcal{I})$ the value of an optimal solution for an instance $\mathcal{I}$ of the $d$-dimensional knapsack problem. We use in the proof of hardness the following parameterized version of the subset sum problem, known as sized subset sum. Given a set of positive integers $L=$ $\left\{x_{1}, \ldots, x_{n}\right\}$, and the positive integer $S, k$, decide if there is a subset $L^{\prime} \subseteq L$ of size $k$, such that the sum of elements in $L^{\prime}$ is exactly $S$ (in this case we say that the input is satisfied). The sized subset sum problem is known to be $W[1]$-hard [4].

We give a reduction from an instance $(L, S, k)$ of sized subset sum to an instance of twodimensional knapsack, denoted by $R(L, S, k)$.

Given an instance $(L, S, k)$, we first modify the values of the elements in $L$. Define

$$
\tilde{x}_{i}=\frac{x_{i}+\frac{k-1}{k} \cdot S}{k},
$$

and let $\tilde{L}=\left\{\tilde{x}_{1}, \ldots, \tilde{x}_{n}\right\}$. Note that, for any $1 \leq i \leq n, 0 \leq \tilde{x}_{i} \leq \frac{2 \cdot S}{k}$ (w.l.o.g. $x_{i} \leq S$ ). An important property of the above transformation is that it does not affect the satisfiability of the original instance.

Lemma 1 The instance $(L, S, k)$ is satisfied if and only if $(\tilde{L}, S, k)$ is satisfied.

Proof: If $(L, S, k)$ is satisfied then there is a subset $\left\{x_{i_{1}}, \ldots, x_{i_{k}}\right\}=L^{\prime} \subseteq L$ such that $\sum_{j=1}^{k} x_{i_{j}}=S$. Consider the subset $\left\{\tilde{x}_{i_{1}}, \ldots, \tilde{x}_{i_{k}}\right\}=\tilde{L}^{\prime} \subseteq \tilde{L}$, then

$$
\sum_{j=1}^{k} \tilde{x}_{i_{j}}=\sum_{j=1}^{k} \frac{x_{i_{j}}+\frac{k-1}{k} \cdot S}{k}=\frac{1}{k} \sum_{j=1}^{k} x_{i_{j}}+\frac{1}{k} \sum_{j=1}^{k} \frac{k-1}{k} \cdot S=S,
$$

\footnotetext{
${ }^{1}$ See also the comprehensive survey of known results in [12].

${ }^{2}$ For the recent theory of fixed-parameter algorithms and parameterized complexity, see, e.g., [5, 3].

${ }^{3}$ The complexity class of SNP was introduced in [15]. The class includes such NP-hard problems as vertex cover, independent set and $3 S A T$, among others. Based on known results in complexity theory, it is unlikely that all of the problems in this class can be solved in sub-exponential time (see [2] and the references therein).
} 
and we have that $(\tilde{L}, S, k)$ is also satisfied.

If $(\tilde{L}, S, k)$ is satisfied, then there is a subset $\left\{\tilde{x}_{i_{1}}, \ldots, \tilde{x}_{i_{k}}\right\}=\tilde{L}^{\prime} \subseteq \tilde{L}$ such that $\sum_{j=1}^{k} \tilde{x}_{i_{j}}=$ $S$. By the definition of $\tilde{L}$, we have that

$$
S=\sum_{j=1}^{k} \tilde{x}_{i_{j}}=\sum_{j=1}^{k} \frac{x_{i_{j}}+\frac{k-1}{k} \cdot S}{k}=\frac{1}{k} \sum_{j=1}^{k} x_{i_{j}}+\frac{1}{k} \sum_{j=1}^{k} \frac{k-1}{k} \cdot S=\frac{1}{k} \sum_{j=1}^{k} x_{i_{j}}+\frac{k-1}{k} \cdot S,
$$

and $\sum_{j=1}^{k} x_{i_{j}}=S$. Thus, $(L, S, k)$ is satisfied as well.

Now, we define the instance $R(L, S, k)$ of two-dimensional knapsack. The items are $\{1, \ldots, n\}$, where each item $i$ has size $\bar{s}_{i}=\left(\tilde{x}_{i}, \frac{2 \cdot S}{k}-\tilde{x}_{i}\right)$ and unit profit. Let $s_{i, 1}$ and $s_{i, 2}$ denote the first and second entries to the vector $\bar{s}_{i}$, respectively. The capacity of the bin is $\bar{B}=(S, S)$. Note that $R(L, S, k)$ can be computed in polynomial time in the size of the instance $(L, S, k)$, and its size is also polynomial.

Lemma $2 \operatorname{OPT}(R(L, S, k)) \leq k$.

Proof: Assume that there is a feasible subset of items $A \subseteq\{1, \ldots, n\}$ whose value is greater than $k$ for $R(L, S, k)$, then $|A| \geq k+1$. Since $A$ is feasible, we have that $\sum_{i \in A} s_{i, 1}=\sum_{i \in A} \tilde{x_{i}} \leq$ $S$, and thus

$$
S \geq \sum_{i \in A} s_{i, 2}=\sum_{i \in A}\left(\frac{2 \cdot S}{k}-\tilde{x}_{i}\right)=|A| \cdot \frac{2 \cdot S}{k}-\sum_{i \in A} \tilde{x}_{i}>S,
$$

a contradiction.

Lemma 3 The instance $(\tilde{L}, S, k)$ is satisfied if and only if $O P T(R(L, S, k)) \geq k$.

Proof: If the instance $(\tilde{L}, S, k)$ is satisfied then there is a subset $\left\{\tilde{x}_{i_{1}}, \ldots, \tilde{x}_{i_{k}}\right\}=\tilde{L}^{\prime} \subseteq \tilde{L}$ such that $\sum_{j=1}^{k} \tilde{x}_{i_{j}}=S$. Thus, the solution $A=\left\{i_{1}, \ldots, i_{k}\right\}$ for $R(L, S, k)$ is feasible in both dimensions, i.e., $\sum_{j=1}^{k} s_{i_{j}, 1}=\sum_{j=1}^{k} \tilde{x}_{i_{j}}=S$, and also $\sum_{j=1}^{k} s_{i_{j}, 2}=\sum_{j=1}^{k}\left(\frac{2 \cdot S}{k}-\tilde{x}_{i_{j}}\right)=S$. The value of this solution is $k$, therefore $\operatorname{OPT}(R(L, S, k)) \geq k$.

If $O P T(R(L, S, k)) \geq k$ then, by Lemma 2, we have that $O P T(R(L, S, k))=k$. Let $A=\left\{i_{1}, \ldots, i_{k}\right\}$ be an optimal solution, then

$$
S \geq \sum_{j=1}^{k} s_{i_{j}, 2}=\sum_{j=1}^{k}\left(\frac{2 \cdot S}{k}-\tilde{x}_{i_{j}}\right)=2 S-\sum_{j=1}^{k} \tilde{x}_{i_{j}}
$$

and we have that $\sum_{j=1}^{k} \tilde{x}_{i_{j}} \geq S$. On the other hand, $S \geq \sum_{j=1}^{k} s_{i_{j}, 1}=\sum_{j=1}^{k} \tilde{x}_{i_{j}}$, and thus $\sum_{j=1}^{k} \tilde{x}_{i_{j}}=S$. It follows that $(\tilde{L}, S, k)$ is satisfied.

By the above discussion, we have the next lemma.

Lemma 4 For any instance $(L, S, k)$ of sized subset sum, $(L, S, k)$ is satisfied if and only if $\operatorname{OPT}(R(L, S, k)) \geq k$.

Proof: The statement of the lemma follows immediately from Lemmas 1 and 3 .

Suppose that we have an approximation scheme $\mathcal{A}(\mathcal{I}, \varepsilon)$ for two-dimensional knapsack. We now show how $\mathcal{A}$ can be used to decide if an input for sized subset sum is satisfied. 
Lemma 5 Let $\mathcal{A}(\mathcal{I}, \varepsilon)$ be an approximation scheme for two-dimensional knapsack with running time $f(1 / \varepsilon) \cdot|\mathcal{I}|^{g(1 / \varepsilon)}$, then there is an algorithm for sized subset sum with running time $f(2 k)$. $|(L, S, k)|^{O(g(2 k))}$.

Proof: Consider the following algorithm for sized subset sum. Given an instance $(L, S, k)$, define the input for two-dimensional knapsack $\mathcal{I}=R(L, S, k)$, and run $\mathcal{A}\left(\mathcal{I}, \frac{1}{2 k}\right)$. If the optimal solution output by the algorithm is of value at least $k$ return that $(L, S, k)$ is satisfied, otherwise return that it cannot be satisfied.

Note that if $O P T(\mathcal{I}) \geq k$, the value output by $\mathcal{A}$ is at least $\left(1-\frac{1}{2 k}\right) k=k-\frac{1}{2}>k-1$. On the other hand, if $O P T(\mathcal{I})<k$, the output value is at most $k-1$. Also, by Lemma $4,(L, S, k)$ is satisfied if and only if $O P T(\mathcal{I}) \geq k$. Hence, the algorithm decides correctly if $(L, S, k)$ is satisfied.

The construction of $\mathcal{I}$ takes polynomial time in $|(L, S, k)|$, and running $\mathcal{A}$ on the instance $\mathcal{I}$ requires $f(2 k) \cdot|R(L, S, k)|^{O(g(2 k))}$ steps. Thus, the running time of the algorithm is $f(2 k)$. $|(L, S, k)|^{O(g(2 k))}$.

We summarize in our main result.

Theorem 6 There is no EPTAS for two-dimensional knapsack unless $W[1]=F P T$.

Proof: Assume there is an EPTAS for two-dimensional knapsack. That is, there exists an algorithm $\mathcal{A}(\mathcal{I}, \varepsilon)$ that, given an instance $\mathcal{I}$ for the problem, returns a $(1-\varepsilon)$-approximation for the optimal solution in $f(1 / \varepsilon) \cdot|\mathcal{I}|^{c}$ steps. Then, by Lemma 5 , there is an algorithm for sized subset sum whose running time is $f(2 k) \cdot|(L, S, k)|^{c^{\prime}}$. It follows that sized subset sum is fixed parameter tractable, which cannot hold unless $W[1]=F P T$.

The standard parametrization of two-dimensional knapsack is as follows. Given an instance of the problem in which all values are integral, and an integer $k \geq 1$, decide if there is a feasible solution of value $k$ or greater. In fact, we have shown the following.

Theorem 7 The standard parametrization of two-dimensional knapsack is W[1]-hard.

We can use the same reduction to derive an explicit lower bound on the running time of approximation schemes for two-dimensional knapsack, under a different complexity measure. To do so, we first derive a lower bound on the complexity of sized subset sum.

Chen at el. show in [2] that unless all problems in SNP are solvable in sub-exponential time, there is no algorithm for independent set whose running time is $f(k) m^{o(k)}$, where $m$ is the input length. Downey and Fellows [4] give a reduction from independent set to perfect code in which, given a graph $G$ and a parameter $k$, a new graph $H$ is constructed, such that $G$ has an independent set of size $k$ iff $H$ has a perfect code of size $k^{\prime}=\frac{k(k+1)}{2}+k+1$. Under the same assumption, this implies that there is no algorithm for perfect code with running time $f(k) m^{o(\sqrt{k})}$, where $m$ is the input size. Furthermore, a reduction given in [4], from perfect code with a parameter $k$ to sized subset sum with the same parameter $k$, implies that there is no algorithm for sized subset sum with running time $f(k) \cdot|\mathcal{I}|^{o(\sqrt{k})}$. This is summarized in the next result.

Lemma 8 Unless all problems in SNP are solvable in sub-exponential time, there is no algorithm for sized subset sum whose running time is $f(k) \cdot|\mathcal{I}|^{o(\sqrt{k})}$, for any function $f$, where $|\mathcal{I}|$ is the input size.

From the above discussion, we have 
Theorem 9 Unless all problems in SNP are solvable in sub-exponential time, there is no approximation scheme for two-dimensional knapsack with running time $f(1 / \varepsilon)|\mathcal{I}|^{o(\sqrt{1 / \varepsilon})}$, for any function $f$, where $|\mathcal{I}|$ is the size of the input for the problem.

Proof: Assume that there is an approximation scheme $\mathcal{A}(\mathcal{I}, \varepsilon)$ for two-dimensional knapsack with running time $f(1 / \varepsilon)|\mathcal{I}|^{o(\sqrt{1 / \varepsilon})}$, for some function $f$. Thus, by Lemma 5 , there is an algorithm for sized subset sum whose running time is $f(2 k)|\mathcal{I}|^{o(\sqrt{k})}$. By Lemma 8, this cannot hold unless all problems in SNP are solvable in sub-exponential time.

In conclusion, we comment that our reductions yield a restricted class of highly structured inputs for $d$-dimensional knapsack, which may not reflect the set of inputs arising in real-life applications. For many inputs, it seems reasonable to assume that a small modification in the bin capacity would result in a small change in the profit of an optimal solution for the given instance. For such inputs, augmenting algorithms, i.e., algorithms that output a solution with profit at least as high as the optimal, while violating the bin capacity (in any dimension) at most by factor $(1+\varepsilon)$, seem to fit well. For fixed values of $d$, an augmenting algorithm, with running time polynomial in $1 / \varepsilon$ and in the input size, can be used to obtain a feasible solution whose profit is at least $1-\varepsilon$ of the optimal. ${ }^{4}$

\section{References}

[1] A. Caprara, H. Kellerer, U. Pferschy, and D. Pisinger. Approximation algorithms for knapsack problems with cardinality constraints. European Journal of Operational Research, 123(2):333 - 345, 2000.

[2] J. Chen, X. Huang, I. A. Kanj, and G. Xia. Linear FPT reductions and computational lower bounds. In STOC '04: Proceedings of the thirty-sixth annual ACM symposium on Theory of computing, pages 212-221, New York, NY, USA, 2004. ACM.

[3] R. G. Downey and M. Fellows. Parameterized Complexity. Monographs in Computer Science. Springer, 1999.

[4] R. G. Downey and M. R. Fellows. Fixed-parameter tractability and completeness II: On completeness for W[1]. Theoretical Computer Science, 141(1-2):109 - 131, 1995.

[5] J. Flum and M. Grohe. Parameterized Complexity Theory (Texts in Theoretical Computer Science. An EATCS Series). Springer-Verlag New York, Inc., Secaucus, NJ, USA, 2006.

[6] A. M. Frieze and M. Clarke. Approximation algorithms for the m-dimensional 0-1 knapsack problem: worst-case and probabilistic analyses. European J. of Operational Research, 15(1):100-109, 1984.

[7] G. Gens and E. Levner. Complexity of approximation algorithms for combinatorial problems: a survey. SIGACT News, 12(3):52-65, 1980.

\footnotetext{
${ }^{4}$ Such an algorithm can be obtained by discretizing the item sizes in each dimension, $r$, to be integral multiples of $\frac{\varepsilon}{n} \cdot B_{r}$, and using dynamic programming over the maximal profit attainable for each of the possible size vectors. Detailed expositions of these standard techniques are given, e.g., in $[8,16]$.
} 
[8] D. S. Hochbaum, editor. Approximation algorithms for NP-hard problems. PWS Publishing Co. Boston, MA, USA, 1996.

[9] K. Jansen. Parameterized approximation scheme for the multiple knapsack problem. In C. Mathieu, editor, Proceedings of the Twentieth Annual ACM-SIAM Symposium on Discrete Algorithms (SODA), pages 665-674. SIAM, 2009.

[10] H. Kellerer and U. Pferschy. A new fully polynomial approximation scheme for the knapsack problem. J. Combinatorial Optimization, 3:59-71, 1999.

[11] H. Kellerer and U. Pferschy. Improved dynamic programming in connection with an FPTAS for the knapsack problem. J. Combinatorial Optimization, 8(1):5-11, 2004.

[12] H. Kellerer, U. Pferschy, and D. Pisinger. Knapsack Problems. Springer, October 2004.

[13] B. Korte and R. Schrader. On the existence of fast approximation schemes. Nonlinear Programming, 4:415-437, 1981.

[14] M. J. Magazine and M.-S. Chern. A note on approximation schemes for multidimensional knapsack problems. Mathematics of Operations Research, 9(2):244-247, 1984.

[15] C. H. Papadimitriou and M. Yannakakis. Optimization, approximation, and complexity classes. J. Comput. Syst. Sci., 43(3):425-440, 1991.

[16] V. Vazirani. Approximation Algorithms. Springer Verlag, 2001. 